\title{
Artificial Neural Network Based Forward Kinematics Solution for Planar Parallel Manipulators Passing through Singular Configuration
}

\author{
Ammar H Elsheikh*, Ezzat A Showaib and Abd Elwahed M Asar \\ Department of Production Engineering and Mechanical Design, Faculty of Engineering, Tanta University, Tanta, Egypt
}

\begin{abstract}
It is well known that, the main drawback of parallel manipulators is the existence of singularities within its workspace, an Artificial Neural Network (ANN) based solution is proposed in this paper. The proposed approach can certainly learn the input-output data and discover the non-linear relationships which are inherent in the training data. Additionally, the proposed approach can provide solution of the forward kinematic problem with reasonable errors at and in the vicinity of kinematic singularities. The approach is implemented for the 3-RPR, 3-PRR, and 3-RRR planar parallel manipulators.
\end{abstract}

Keywords: Parallel manipulators; Forward kinematics; Singularities; Artificial Neural Network (ANN)

\section{Introduction}

Parallel manipulators, due to its closed-loop structure, posses a number of advantages over traditional serial manipulators such as high rigidity, high load-to-weight ratio, high natural frequencies, high speed and high accuracy [1]. However, they also have a few disadvantages such as a relatively small workspace, relatively complex forward kinematics and the most importantly, existence of singularities inside the workspace [2]. Kinematics analysis of parallel manipulators separate in two types, forward kinematics and inverse kinematics. The inverse kinematics, which maps the task space to joint space, is not difficult to solve. On the other hand, the forward kinematics, which maps the joint space to task space, is so hard to solve. Also, the existence of not only multiple inverse kinematic solutions (or working modes) but also multiple forward kinematic solutions (or assembly modes) is another problem in kinematics analysis [3]. The challenging problem is not to find all possible solutions but to directly determine the unique feasible solutions, the actual physical solution, in among all possible solutions starting from a certain initial configuration [4].

Forward kinematics and singularity analysis of planar parallel manipulators have been investigated by many researchers [5-7]. Efforts to solve the forward kinematics of planar parallel manipulators have concentrated on 3-RPR manipulator due to its inherent simplicity. It is established the forward kinematic solution of general 3 DOF planar parallel manipulators can be lead to a polynomial of degree 8 [8]. However, the forward kinematic problem for the manipulator under study leads to a maximum of 6 real solutions. It is worth taking into considerations, the three manipulators under study are kinematically equivalent to each other and, as a result, we derived the forward kinematics equations for 3-RRR and modified it to the two other manipulators. Additionally, the existence of singularities and uncertainties inside the workspace where the manipulator gains some degrees of freedom and become uncountable. In such configurations, the actuated joints forces of the manipulator will become unacceptably large that often reach their allowable limits. To overcome the problem of kinematics singularities a neural network -based approach is developed which has the ability of generalization and can successfully learn relationships that are not present in the training set in an efficient manner.

There have been increasing research interests of Artificial Neural
Networks (ANNs) due to their extreme flexibility and the capability of non-linear function approximation. Many efforts have been made on applications of Neural Networks to various types of parallel manipulators [9-13].

In this paper, a supervised neural network approach is developed to control the motion of the 3-RPR, 3-PRR and 3-RRR planar parallel manipulators. Multiple neural networks are used to overcome the problem of the multiple solution branches of either forward or inverse kinematics. This approach also overcomes the problems of singularities and uncertainties' arising in trajectory planning as it has, like any ANN algorithms, generalization ability. In this approach a network is trained using training data generated from the inverse kinematics. The training is done off-line until reaching acceptable error and a validation test is also done, at each iteration, to avoid model over fitting. It may be noted here that the present work may be considered as an implementation of the artificial neural network approach for serial manipulators passing through singular configuration, as proposed by [14], for planar parallel manipulators.

\section{Kinematics of Parallel Manipulators}

Kinematic analysis of parallel manipulators includes solution to forward and inverse kinematic problems. The forward kinematics of a manipulator deals with the computation of the position and orientation of the manipulator end-effector in terms of the active joints variables. Forward kinematic analysis is one of essential parts in control and simulation of parallel manipulators. Contrary to the forward kinematics, the inverse kinematics problem deal with the determination of the joint variables corresponding to any specified position and orientation of the end-effector. The inverse kinematics problem is

*Corresponding author: Ammar $\mathrm{H}$. Elsheikh, Department of Production Engineering and Mechanical Design, Faculty of Engineering, Tanta University, Tanta, Egypt, E-mail: eng_ammar_sheikh@yahoo.com

Received June 30, 2013; Accepted September 18, 2013; Published September 20, 2013

Citation: Elsheikh AH, Showaib EA, Asar AE (2013) Artificial Neural Network Based Forward Kinematics Solution for Planar Parallel Manipulators Passing through Singular Configuration. Adv Robot Autom 2: 106. doi: 10.4172/21689695.1000106

Copyright: (c) 2013 Elsheikh AH, et al. This is an open-access article distributed under the terms of the Creative Commons Attribution License, which permits unrestricted use, distribution, and reproduction in any medium, provided the original author and source are credited. 
essential to execute manipulation tasks. Most parallel manipulators can admit not only multiple inverse kinematic solutions, but also multiple forward kinematic solutions. This property produces more complicated kinematic models but allows more flexibility in trajectory planning [15]. In other words, a manipulator configuration can be defined either by actuator coordinates $\mathrm{q}=\left[\mathrm{q}_{1}, . ., \mathrm{q}_{\mathrm{n}}\right]^{\mathrm{T}}$ or by Cartesian end-effector coordinates $\mathrm{x}=\left[\mathrm{x}_{1}, \ldots, \mathrm{x}_{\mathrm{n}}\right]^{\mathrm{T}}$ with $\mathrm{n}$ the DOF of the manipulator under study. The transformation between actuator coordinates and Cartesian coordinates is an important issue from viewpoint of kinematic control. Computation of the end-effector coordinates from given actuator coordinates (forward kinematics) can be written in the general form

$$
\mathrm{X}=f_{\text {FKP }}(\mathrm{q})
$$

The inverse task which is to establish the actuator coordinates corresponding to a given set of end effector coordinates (inverse kinematics) can be also written in the general form

$$
\mathrm{q}=f_{\text {IKP }}(\mathrm{x})
$$

Then the kinematic constraints imposed by the limbs can be written in the general form

$$
f(\mathrm{x}, \mathrm{q})=0
$$

Differentiating Eq.(3) with respect to time, we obtain a relationship between the input joint rates and the end-effector output velocity

$$
J_{x} \dot{x}=J_{q} \dot{q}
$$

Where

$$
\mathrm{J}_{\mathrm{x}}=\frac{\partial f}{\partial x} \text { and } \mathrm{J}_{\mathrm{q}}=\frac{\partial f}{\partial q}
$$

Inverse kinematic singularity occurs when different inverse kinematic solutions coincide that happens usually at the workspace boundary. Hence the manipulator loses one or more degrees of freedom. Mathematically they can detected by $\operatorname{det}\left(\mathrm{J}_{\mathrm{q}}\right)=0$

Forward kinematic singularity occurs when different forward kinematic solutions coincide. Hence the manipulator gains one or more degrees of freedom. That happens inside the workspace so it is a great problem. Mathematically they can detected by $\operatorname{det}\left(J_{\mathrm{x}}\right)=0$

\section{Manipulators Under Study}

The architectures of the planar parallel manipulators under study, 3-RPR, 3-PRR and 3-RRR, are illustrated in Figures 1a-1c, Where R, $\mathrm{P}, \mathrm{R}$ and $\mathrm{P}$ denote revolute, prismatic, actuated revolute and actuated prismatic joints, respectively. For manipulators under study the three fixed pivots $A_{1}, A_{2}$ and $A_{3}$ define the geometry of the fixed base, and the three moving pivots $\mathrm{C}_{1}, \mathrm{C}_{2}$ and $\mathrm{C}_{3}$ define the geometry of the moving platform, where point $\mathrm{O}$ and $\mathrm{H}$ are the centroids of the fixed base and moving platform respectively. Three limbs connect the moving platform to the fixed base. Each limb of the 3-RPR is composed of a R, a P, and a

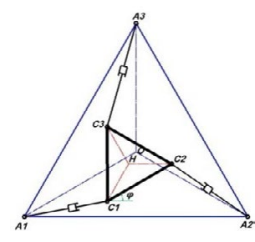

(a) 3-RPR

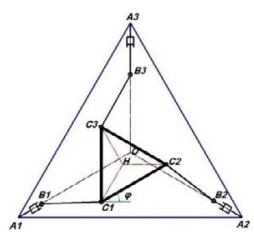

(b) 3-PRR

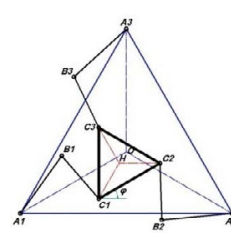

(c) 3-RRR
Figure 1: The three planar parallel manipulators under study.

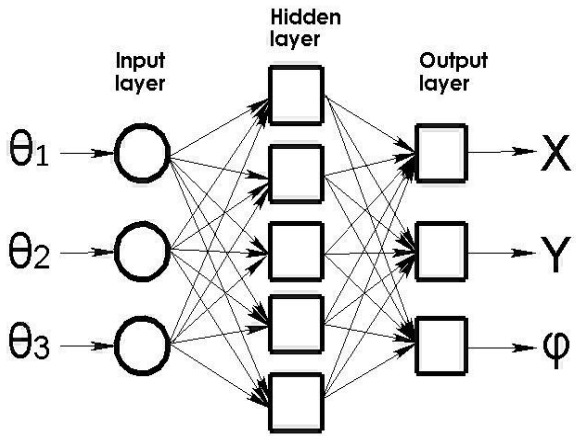

Figure 2: The topology of MLP network with a single hidden layer.

$R$ joint in sequence. Each limb of the 3-PRR is composed of a $P$, a R, and a $R$ joint in sequence. Likewise, each limb of the $3-R R R$ is composed of three $\mathrm{R}$ joints in sequence. The origin of the fixed coordinate frame is located at point $A_{1}$. The $x$-axis points along the direction of $A_{1} A_{2}$ and the $y$-axis is perpendicular to $\mathrm{A}_{1} \mathrm{~A}_{2}$. We assume that the manipulators under study are symmetrical, manipulators with equilateral base and moving platform [15]. The moving platform pose, i.e., its position and its orientation, is determined by means of the Cartesian coordinates vector $\mathrm{H}=\left[\mathrm{H}_{\mathrm{x}}, \mathrm{H}_{\mathrm{y}}\right]^{\mathrm{T}}$ of operation point $\mathrm{H}$ and angle $\varphi$, namely, the angle between $\mathrm{C}_{1} \mathrm{C}_{2}$ and the positive direction of $\mathrm{x}$-axis.

\section{Artificial Neural Networks}

Artificial neural network (ANN) is an algorithm that model brain performs a particular task, and is usually implemented using electronic components or simulated in software on digital computers. It has the ability of imitating of the mechanisms of learning and problem solving functions of the human brain which are flexible, powerful, and robust. In artificial neural networks implementation, knowledge is represented as numeric weights, which are used to gather the relationships between data that are difficult to realize analytically, and this iteratively adjusts the network parameters to minimize the sum of the squared approximation errors using a gradient descent method [14]. One category of the artificial neural networks is the multilayer perceptron (MLP) which be considered a supervised back propagation learning algorithm. It consists of an input layer, some hidden layers and an output layer as shown in Figure 2. MLP is trained by back propagation of errors between desired values and outputs of the network using some effective algorithms such as gradient descent algorithm. The network starts training after the weight factors are initialized randomly. Weight adjusting takes place until, we get reasonable errors or no more weight changes occur. There is no available theoretical procedures to choose the appreciate network architecture, i.e. number of hidden layers and number of neurons of each layer. This depends on the problem under investigation and user's experience.

\section{Results of Numerical Simulations}

Simulations have been conducted for the 3-RPR, 3-PRR, and 3-RRR planar parallel manipulators to demonstrate the performance of the developed approach. First point $\mathrm{H}$ (the centroid of the end-effector) is moved along a given trajectory which passing through singular locus then the correct active prismatic joint or joint angle variables to track this trajectory are calculated using the inverse kinematic model of the simulated manipulator which give a unique solution for a given working mode. Then, those active prismatic joint or joint angle variables are fed 


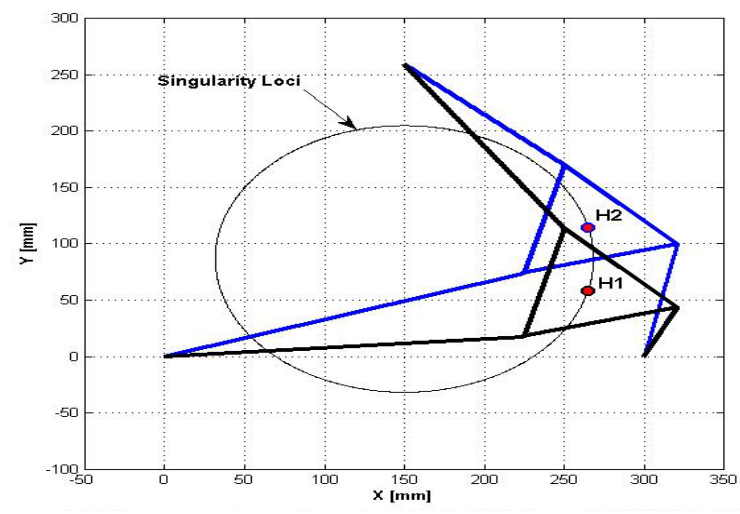

Figure 3: The 3-RPR cross singularity loci at $\mathrm{H} 1(265,58.499)$ and $\mathrm{H} 2(265,114.706)$.

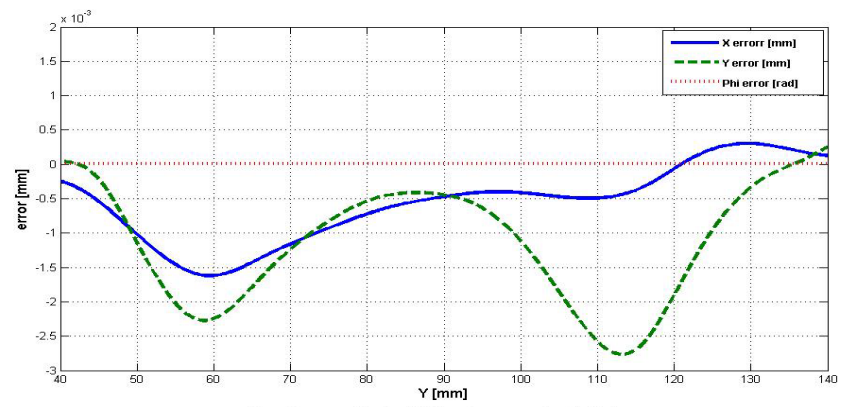

Figure 4: Tracking error for the 3-RPR along a vertical trajectory passing through singularity points $\mathrm{H} 1(265,58.499)$ and $\mathrm{H} 2(265,114.706)$.

to the MIP to track the trajectory and the tracking errors are calculated. The simulated manipulators are assumed to be ideal mechanisms with no flexibility and no joint clearance that affect the accuracy of the manipulator. Also, the prismatic joints are assumed to have unlimited length. A two-hidden layer MLP with back propagation learning is considered here. The input layer has as many nodes as the number of inputs to the map, namely three actuator variables. Similarly the output layer will have three nodes which represent the pose of the end-effector. The number of neurons in the hidden layers and its configuration are used as a design parameter. Sigmoid and linear activation functions are used for all hidden and output layer nodes respectively. Supervised learning scheme is used in which the network is taught to learn the map by observing the inputs and outputs. The network is trained by 10,000 training input-output patterns generated, randomly within the workspace of the manipulator, from the inverse kinematic model. Random initialization is used for the weights. For each manipulator, different configurations of the MLP network were tested to get the optimal configuration used for solve the problem. About 36 multi-layer feed forward networks with two hidden layers are trained. All these networks were trained over 1,000 training epochs to ensure the success of the training process and to avoid over fitting the model. Simulation results showed that $40 \times 60$ multilayer perceptron neural network with two hidden layers had the best performance when the minimum tracking error is used as performance index. All manipulators under study are symmetric with three identical limbs. Each side of the moving end-effector equilateral triangle is $100 \mathrm{~mm}$, while that of the base is 300 $\mathrm{mm}$. The lengths of the proximal links and the distal links are $120 \mathrm{~mm}$ and $80 \mathrm{~mm}$, respectively.

\section{RPR planar parallel manipulator}

Three end-effector trajectories are specified as straight lines which cross over singularity loci at $\mathrm{H} 1(265,58.499) \mathrm{mm}$ and $\mathrm{H} 2(265,114.706)$ $\mathrm{mm}$ as shown in Figure 3. The first trajectory is a vertical straight line starting at $\mathrm{H}_{i}(265,40) \mathrm{mm}$ with orientation angle $\varphi=15^{\circ}$ and ending at point $\mathrm{H}_{\mathrm{f}}(265,140) \mathrm{mm}$ with the same orientation it is obvious the selected trajectory passes through singular points $\mathrm{H} 1$ and $\mathrm{H} 2$. The tracking errors in $\mathrm{x}$ - and $\mathrm{y}$-directions are depicted in Figure 4 . The maximum tracking error along the trajectory points is $0.0027 \mathrm{~mm}$ which happens in the vicinity of kinematic singularities. We also note that there is a significant increasing in the tracking error near the singularity points. Anyway, the developed approach can provide solution for the problem with reasonable errors.

The second trajectory is a horizontal straight lines starting at $\mathrm{H}_{\mathrm{i}}(245,58.499) \mathrm{mm}$ with orientation angle $\varphi=15^{\circ}$ and ending at point $\mathrm{H}_{\mathrm{f}}(275,58.499) \mathrm{mm}$ with the same orientation it is obvious the selected trajectory passes through singular point $\mathrm{H} 1$. The tracking errors in $\mathrm{x}$ and y-directions are depicted in Figure 5. The maximum tracking error

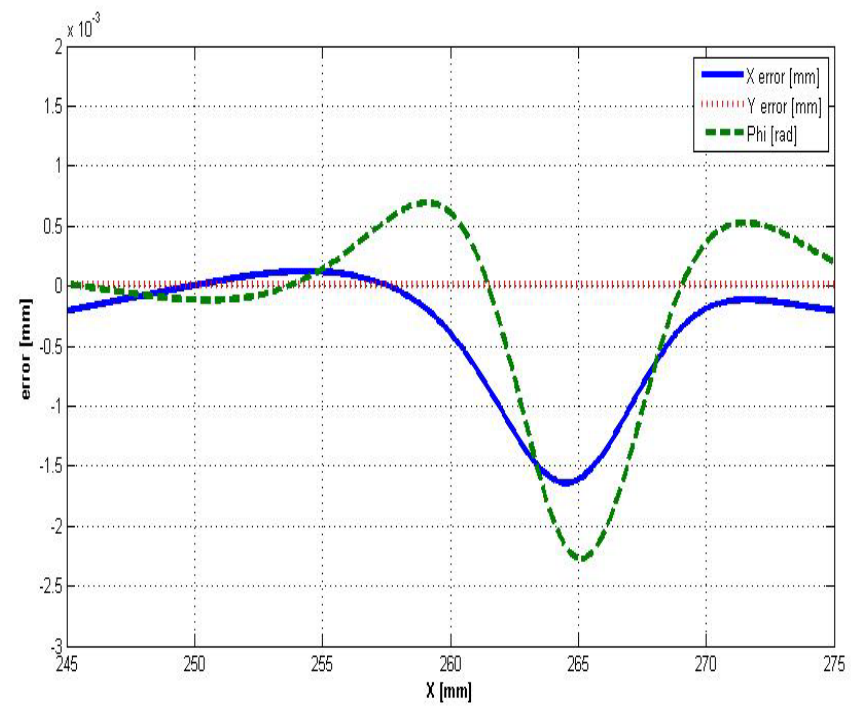

Figure 5: Tracking error for the 3-RPR along a horizontal trajectory passing through singularity point $\mathrm{H} 1(265,58.499)$.

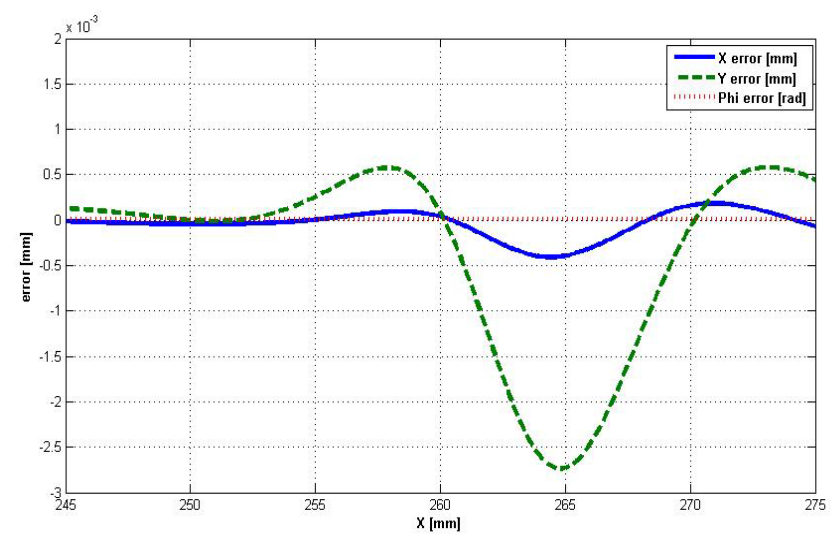

Figure 6: Tracking error for the 3-RPR along a horizontal trajectory passing through singularity point $\mathrm{H} 1(265,58.499)$. 


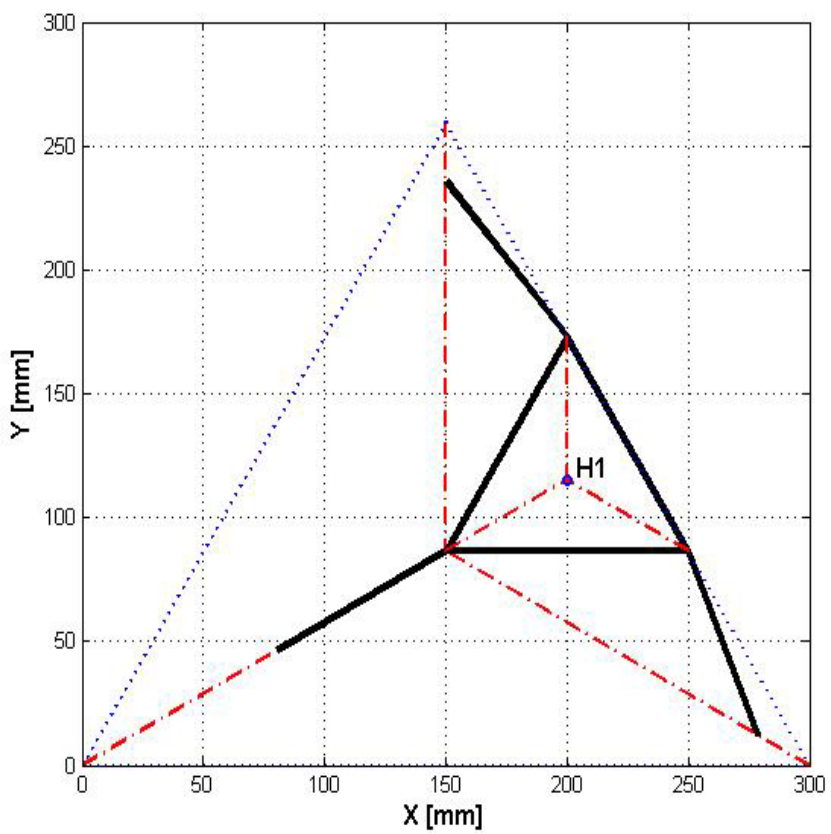

Figure 7: The 3-PRR cross singularity loci at $\mathrm{H} 1(200,115.47)$.

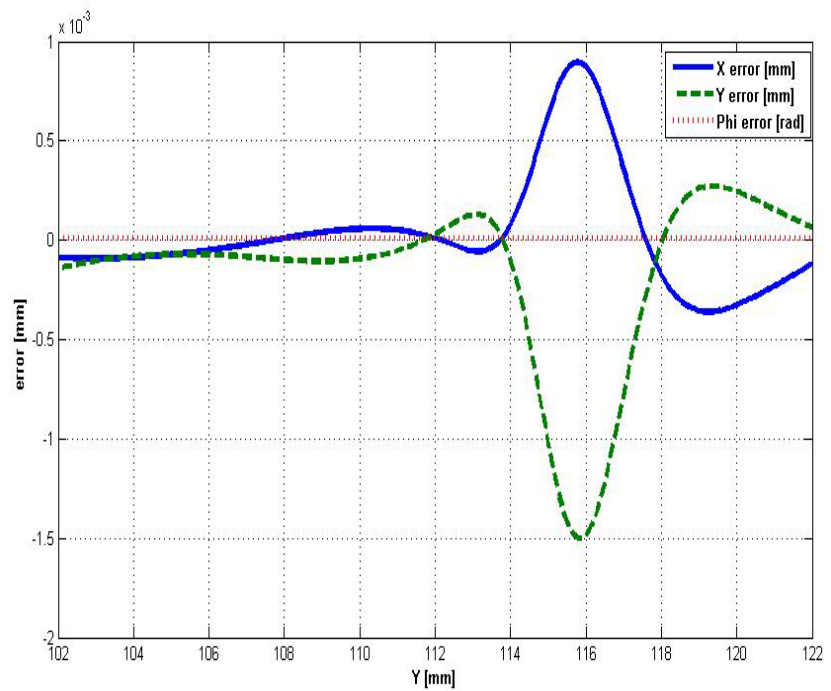

Figure 8:Tracking error for the 3-PRR along a vertical trajectory passing through singularity point $\mathrm{H} 1(200,115.470)$.

along the trajectory points is $0.0023 \mathrm{~mm}$ which also happens in the vicinity of kinematic singularities.

Finally, the third trajectory is a horizontal straight lines starting at $\mathrm{H}_{\mathrm{i}}(245,114.706) \mathrm{mm}$ with orientation angle $\varphi=15^{\circ}$ and ending at point $\mathrm{H}_{\mathrm{f}}(275,114.706) \mathrm{mm}$ with the same orientation. The selected trajectory passes through singular point $\mathrm{H} 2$. The tracking errors in $\mathrm{x}$ and $y$-directions are depicted in Figure 6. The maximum tracking error along the trajectory points is $0.0027 \mathrm{~mm}$ which also happens in the vicinity of kinematic singularities.

\section{PRR planar parallel manipulator}

Two end-effector trajectories are specified as straight lines which cross over singularity loci at $\mathrm{H}_{1}(200,115.470) \mathrm{mm}$ as shown in Figure 7 . The first trajectory is a vertical straight line starting at $\mathrm{H}_{\mathrm{i}}(200,102) \mathrm{mm}$ with orientation angle $\varphi=0^{\circ}$ and ending at point $\mathrm{H}_{f}(200,122) \mathrm{mm}$ with the same orientation it is obvious the selected trajectory passes through singular point $\mathrm{H} 1$. The tracking errors in $\mathrm{x}$ - and $\mathrm{y}$-directions are depicted in Figure 8. The maximum tracking error along the trajectory points is $0.0015 \mathrm{~mm}$ which happens in the vicinity of kinematic singularities.

The second trajectory is a horizontal straight lines starting at

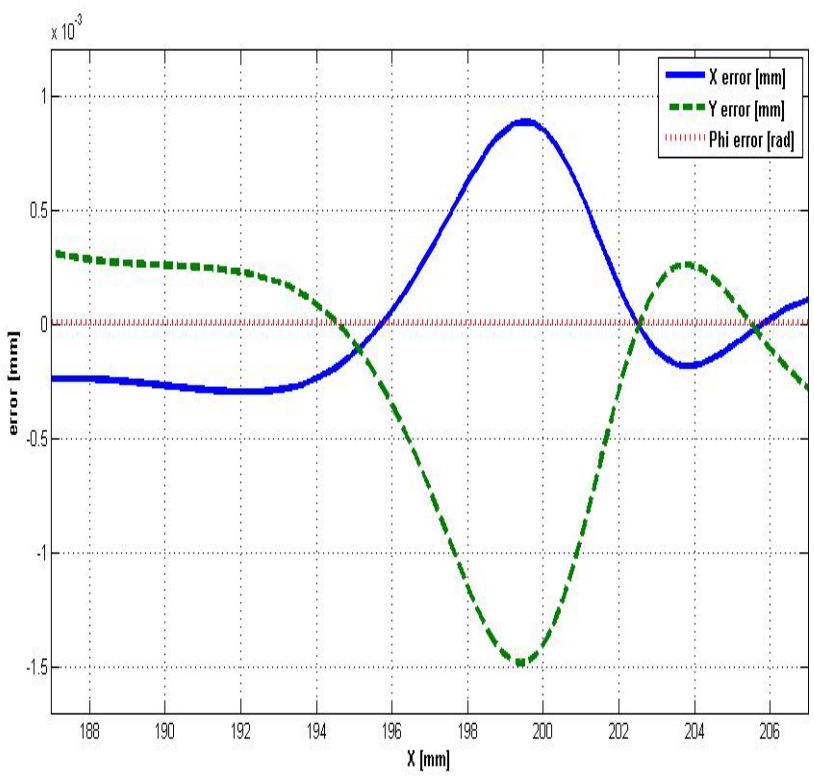

Figure 9: Tracking error for the 3-PRR along a horizontal trajectory passing through singularity point $\mathrm{H} 1(200,115.470)$.

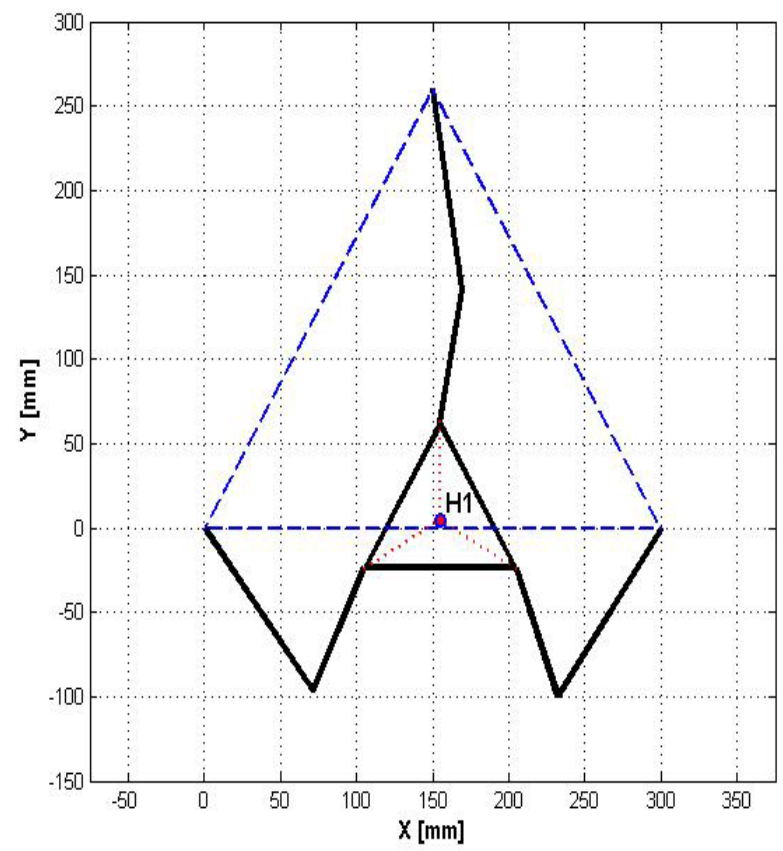

Figure 10: The 3-RRR cross singularity loci at $\mathrm{H} 1(155,4.845)$. 


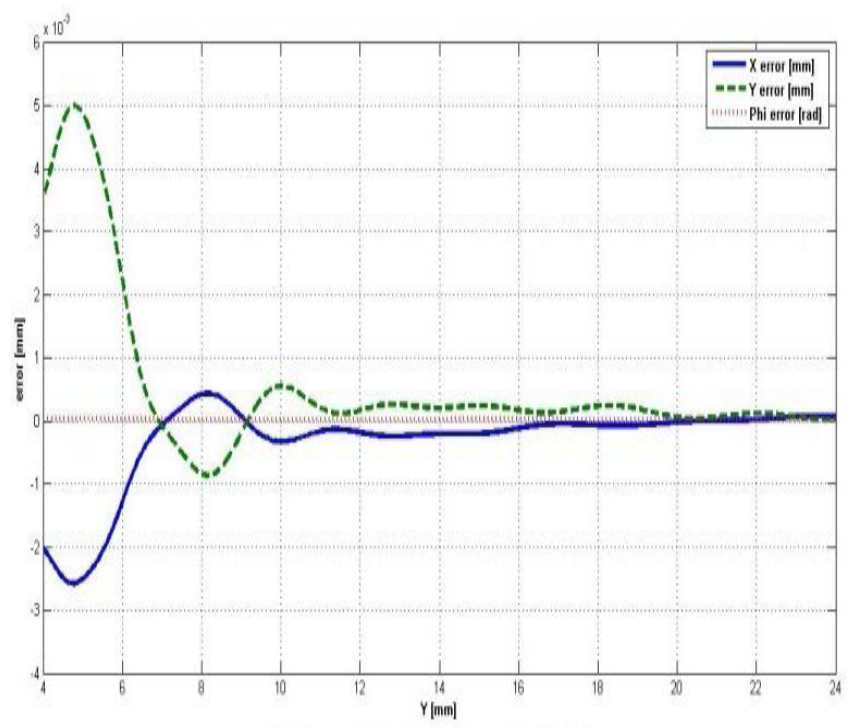

Figure 11: Tracking error for the 3-RRR along a vertical trajectory passing through singularity point $\mathrm{H} 1(155,4.845)$.

$\operatorname{Hi}(187,115.470) \mathrm{mm}$ with orientation angle $\varphi=0^{\circ}$ and ending at point $\operatorname{Hf}(207,115.470) \mathrm{mm}$ with the same orientation it is obvious the selected trajectory passes through singular point $\mathrm{H} 1$. The tracking errors in $\mathrm{x}$ and y-directions are depicted in Figure 9. The maximum tracking error along the trajectory points is $0.0015 \mathrm{~mm}$ which also happens in the vicinity of kinematic singularities.

\section{RRR planar parallel manipulator}

In the same way, two end-effector trajectories are specified as straight lines which cross over singularity loci at $\mathrm{H}_{1}(155,4.845) \mathrm{mm}$ as shown in Figure 10. The first trajectory is a vertical straight line starting at $\operatorname{Hi}(155,4) \mathrm{mm}$ with orientation angle $\varphi=0^{\circ}$ and ending at point $\mathrm{H}_{\mathrm{f}}(155,24) \mathrm{mm}$ with the same orientation it is obvious the selected trajectory passes through singular point $\mathrm{H}_{1}$. The tracking errors in $\mathrm{x}$ and $y$-directions are depicted in Figure 11. The maximum tracking error along the trajectory points is $0.005 \mathrm{~mm}$ which happens in the vicinity of kinematic singularities.

The second trajectory is a horizontal straight lines starting at $\mathrm{H}_{\mathrm{i}}(142,4.845) \mathrm{mm}$ with orientation angle $\varphi=0^{\circ}$ and ending at point $\mathrm{H}_{\mathrm{f}}(162,4.845) \mathrm{mm}$ with the same orientation it is obvious the selected trajectory passes through singular point $\mathrm{H}_{1}$. The tracking errors in $\mathrm{x}$ and $y$-directions are depicted in Figure 12. The maximum tracking error along the trajectory points is $0.0053 \mathrm{~mm}$ which also happens in the vicinity of kinematic singularities.

\section{Conclusion}

In this paper, we proposed to use neural networks for forward kinematic solution of three different architectures of planar parallel manipulators, which can be elaborated to generate the best estimation of forward kinematics of the manipulators under study. Even though the manipulators passing through the kinemaic singularities, the proposed approach can provide solution for the problem with reasonable errors. The results of this paper can be used to find the forward kinematics solutions at critical points (singularity points) which can be then avoided, as long as we specify them, in dynamic control stage.

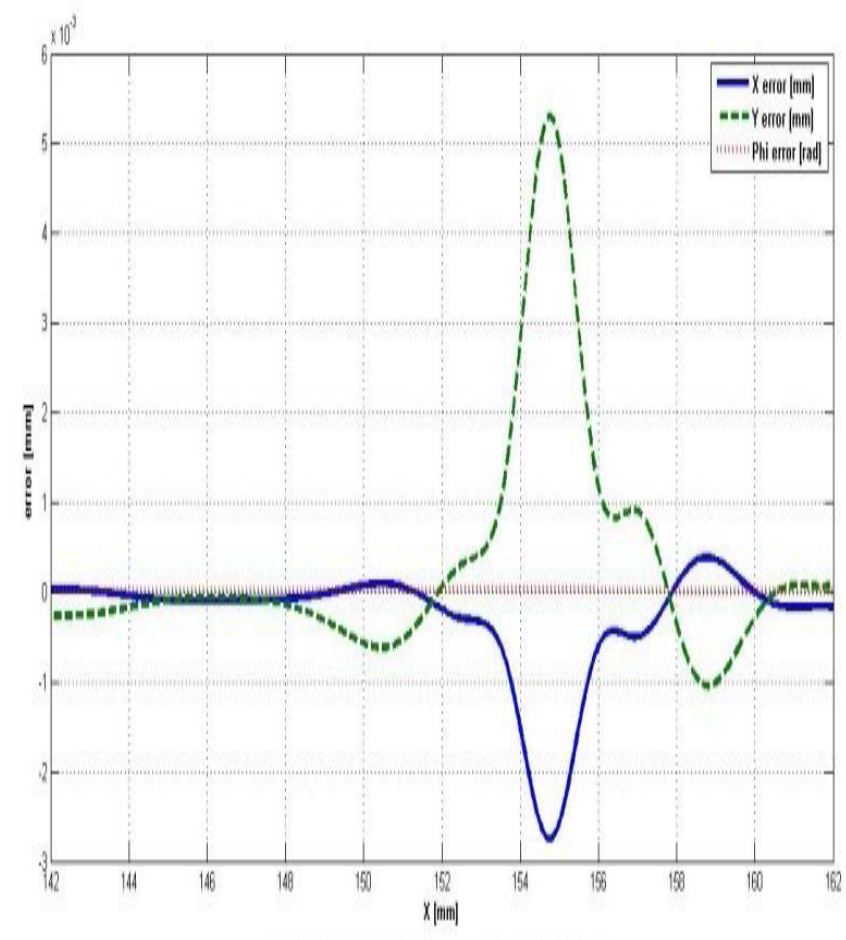

Figure 12: Tracking error for the $3-R R R$ along a horizontal trajectory passing through singularity point $\mathrm{H} 1(155,4.845)$.

\section{References}

1. Cheng H, Liu GF, Yiu YK, Xiong ZH, Li ZX (2001) Advantages and dynamics of parallel manipulators with redundant actuation. IEEE/RSJ International Conference on Intelligent Robots and Systems Proceedings, $\mathrm{HI}$.

2. Hunt $\mathrm{KH}(1983)$ Structural Kinematics of In-Parallel-Actuated Robot-Arms. J. Mech. Des 105:705-712.

3. Merlet JP(2006) Parallel Robots. Kluwer Academic, Dordrecht, The Netherlands.

4. Samy FM Assal (2012) Self-organizing approach for learning the forward kinematic multiple solutions of parallel manipulators. Robotica 30: 951-961.

5. Gosselin CM, Sefrioui J (1991) Polynomial solutions for the direct kinematic problem of planar three-degree-of-freedom parallel manipulators. ICAR Fifth International Conference on Robots in Unstructured Environments, Italy.

6. Merlet JP (1996) Direct kinematics of planar parallel manipulators. IEEE International Conference on Robotics and Automation, France.

7. Oetomo D, Hwee C L, Alici G, Shirinzadeh B(2006) Direct Kinematics and Analytical Solution to 3RRR Parallel Planar Mechanisms.ICARCV '06. 9th International Conference on Control, Automation, Robotics and Vision, Singapore.

8. Tsai LW (1999) Robot Analysis: The Mechanics of Serial and Parallel Manipulators.

9. Yee CS, Kah-Bin Lim (1991) Neural network for the forward kinematics problem in parallel manipulator. IEEE International Joint Conference on Neural Networks.

10. Dehghani M, Ahmadi M, Khayatian A, Eghtesad, M (2008) Wavelet Based Neural Network Solution for Forward Kinematics Problem of HEXA Parallel Robot INES International Conference on Intelligent Engineering Systems, Iran.

11. Lee Hyung Sang, Myung-Chul Han (1991) The estimation for forward kinematic solution of Stewart platform using the neural network IROS '99 IEEE/RSJ International Conference on Intelligent Robots and Systems, Kyongju.

12. Parikh PJ, Lam SSY (2005) A hybrid strategy to solve the forward kinematics problem in parallel manipulators IEEE Transactions on Robotics. 
Citation: Elsheikh AH, Showaib EA, Asar AE (2013) Artificial Neural Network Based Forward Kinematics Solution for Planar Parallel Manipulators Passing through Singular Configuration. Adv Robot Autom 2: 106. doi: 10.4172/2168-9695.1000106

Page 6 of 6

13. Dan Zhang, Jianhe Lei (2011) Kinematic analysis of a novel 3-DOF actuation redundant parallel manipulator using artificial intelligence approach. Robotics and Computer-Integrated Manufacturing 27: 157-163.

14. Ali TH, Ismail N Hamouda AMS, Ishak Aris, Marhaban MH et.al (2010) Artificial neural network-based kinematics Jacobian solution for serial manipulator passing through singular configurations. Advances in Engineering Software 41 359-367.

15. Wenger P, Chablat D (2004) The Kinematic Analysis of a Symmetrical ThreeDegree-of-Freedom Planar Parallel Manipulator. CISM-IFTOMM Symposium on Robot Design, Dynamics and Control Montreal. 